\title{
LAMC1 is related to the poor prognosis of patients with gastric cancer and facilitates cancer cell malignancies
}

\author{
Zhi-Rong HAN $^{*}$, Xiao-Lin JIANG, Wen-Chuan FAN \\ Department of Pathology, Guangyuan Central Hospital, Guangyuan, Sichuan, China \\ *Correspondence: SDFio67gh@163.com
}

Received November 17, 2020 / Accepted February 11, 2021

\begin{abstract}
Gastric cancer is a common malignancy in the alimentary system. The laminin subunit gamma 1 (LAMC1) is an important oncogene in human cancers. However, how and whether LAMC1 takes part in gastric cancer progression is largely uncertain. This study analyzed the association between clinical factors of patients and LAMC1 expression and explored the influence of LAMC1 silencing on cell proliferation, migration, invasion, the Warburg effect, protein kinase B (AKT) pathway, and mitogen-activated protein kinase (MEK)/extracellular regulated protein kinase (ERK) pathway in gastric cancer cells. Our results showed LAMC1 abundance was enhanced in gastric cancer samples and cells. LAMC1 was related to the clinical stage, tumor depth, lymph node metastasis, and distant metastasis of patients. LAMC1 silencing inhibited cell proliferation, migration, and invasion. Moreover, LAMC1 knockdown suppressed the Warburg effect via decreasing lactate production, lactate dehydrogenase (LDH) activity, and glucose uptake. LAMC1 interference blocked the activation of the AKT and MEK/ERK signaling. Collectively, LAMC1 knockdown constrained cell proliferation, migration, invasion, and the Warburg effect in gastric cancer cells via inactivating the AKT and MEK/ERK pathway.
\end{abstract}

Key words: gastric cancer, LAMC1, proliferation, Warburg effect, AKT, MEK/ERK pathway

Gastric cancer is common cancer with over 1 million new cases and about 784,000 deaths globally [1]. The infection of Helicobacter pylori is the major risk for gastric cancer [2]. In recent years, the outcome of gastric cancer therapy has gained many advances [3], however, the prognosis is still poor in patients at an advanced stage [4]. Thus, it is hoped to determine novel strategies including identifying new biomarkers for the targeted therapy of gastric cancer.

A laminin family is a group of the most widely expressed extracellular matrix proteins, mainly including five alpha, four beta, and three gamma variants, which exert important roles in cell processes [5]. For instance, the laminin alpha 4, beta 3 , and gamma 2 have been reported to be tightly linked to the malignancy of gastric cancer $[6,7]$. The laminin subunit gamma 1 (LAMC1) is an important prognostic factor in human cancers, like hepatocellular carcinoma and endometrial cancer $[8,9]$. Additionally, LAMC1 facilitated prostate cancer cell migration and invasion [10]. Moreover, LAMC1 contributed to cell proliferation and the Warburg effect in hepatocellular carcinoma via activating the protein kinase B (AKT) pathway [11]. However, it is unclear whether LAMC1 could take part in the progression of gastric cancer.
In this study, we mainly focused on the correlation between LAMC1 expression and clinal characteristics of gastric cancer patients, and the effects of LAMC1 knockdown on gastric cancer cell malignancy. Besides, the downstream pathway of LAMC1 was further investigated to figure out the molecular basis of LAMC1. Our study might imply a crucial role of LAMC1 in gastric cancer prognosis and therapy.

\section{Patients and methods}

Bioinformatics analysis. LAMC1-related genes were predicted using an online TCGA data processing tool, LinkedOmics (https://www.linkedomics.org/login.php) [12]. Total of 888 genes were selected with an adjusted $\mathrm{p}<0.05$ and correlation coefficient $|\mathrm{r}|>0.5$. Kyoto Encyclopedia of Genes and Genomes (KEGG) analysis, a database with information on biological pathways [13], was employed to analyze the important signaling pathways involved in the regulatory network of LAMC1 using DAVID 6.8 (https:// david.ncifcrf.gov) [14].

Patients and tissue collection. The patients with gastric cancer $(n=42)$ were recruited from Guangyuan Central 
Hospital, without anti-tumor therapy prior to the surgery. The paired tumor and normal samples $(5 \mathrm{~cm}$ away from tumor edge) were collected during surgery and maintained in liquid nitrogen. The clinical data of patients were recorded, and the association between the clinical formation and LAMC1 was analyzed in Table 1 . The written informed content was obtained from every patient who was informed of the usage and purpose of the study. This research was authorized by the ethical approval of Guangyuan Central Hospital.

Immunohistochemistry (IHC) and fluorescence in situ hybridization (FISH) analyses. LAMC1 in tissues was detected via IHC and FISH analyses. For IHC analysis, the paraffin-embedded tumor or normal tissues were cut into $4 \mu \mathrm{m}$ sections. The sections were baked at $65^{\circ} \mathrm{C}$ for $2 \mathrm{~h}$, deparaffinized in xylene, rehydrated in gradient ethanol, and blocked in 3\% hydrogen peroxide. Next, the sections were incubated with anti-LAMC1 (ab233389, 1:1,000 dilution; Abcam, Cambridge, UK) overnight and horseradish peroxidase (HRP)-labeled IgG (ab97051, 1:1,000 dilution; Abcam) for $1 \mathrm{~h}$. Then sections were dyed with 3,3-diaminobenzidine (DAB) (Solarbio, Beijing, China) for $2 \mathrm{~min}$, and the nuclei were stained with hematoxylin (Solarbio). After the dehydration, sections were observed under a microscope (Olympus, Tokyo, Japan).

For FISH analysis, the probes targeting LAMC1 were constructed using FISH Tag RNA Rad kit (Thermo Fisher Scientific, Waltham, MA, USA), which were labeled with Alexa Fluor 594 dye. The sections were incubated with the probes at $37^{\circ} \mathrm{C}$ for $16 \mathrm{~h}$, and the nuclei were stained by 4',6-diamidino-2-phenylindole (DAPI) (Solarbio). The sections were visualized under a fluorescence microscope (Olympus).

Cell culture. Four gastric cancer cell lines (AGS, MKN45, SNU-1, and SNU16) and human gastric epithelial cell line (GES-1) were purchased from Procell (Wuhan, China) or ATCC (Manassas, VA, USA), and maintained in RPMI-1640 medium (Solarbio) plus 10\% FBS (Gibco, Grand Island, NY, USA) and $1 \%$ penicillin/streptomycin (Gibco) in $5 \% \mathrm{CO}_{2}$ and $37^{\circ} \mathrm{C}$.

Cell transfection. The short hairpin RNA (shRNA) for LAMC1 knockdown (shLAMC1-1, 5'-AAAAAUCCAGGUUUGCAUCUU-3'; shLAMC1-2, 5'-UCAUCAAUCUGAAAGGUAGAG-3'; shLAMC1-3, 5'-UAUGUUAUUUGCUUCAUUCUC-3') and negative control (shNC, 5'AAGACAUUGUGUGUCCGCCTT-3') were generated by GenePharma (Shanghai, China). AGS and MKN45 cells with $60-70 \%$ confluences were transfected with $20 \mathrm{nM}$ oligonucleotides using Lipofectamine 2000 (Thermo Fisher Scientific) following the instructions. After the transfection for $24 \mathrm{~h}$, the LAMC1 abundance was measured to evaluate the knockdown efficiency.

Quantitative reverse transcription polymerase chain reaction (qRT-PCR). The tissue samples or cells were lysed in Trizol (Thermo Fisher Scientific) for RNA isolation according to the instructions. The complementary
DNA (cDNA) was generated from $800 \mathrm{ng}$ RNA with the M-MLV reverse transcription kit (Thermo Fisher Scientific). The cDNA was mixed with SYBR (Vazyme, Nanjing, China) and primer pairs, and then used for qRT-PCR with an amplification protocol: $94^{\circ} \mathrm{C}$ for $5 \mathrm{~min}, 35$ cycles of $94^{\circ} \mathrm{C}$ for $20 \mathrm{~s}$, and $60^{\circ} \mathrm{C}$ for $1 \mathrm{~min}$, followed by $72^{\circ} \mathrm{C}$ for 5 $\min$. The sequences for primer pairs were listed as: LAMC1 (sense: 5'-CTTCTGAGGACACTGGCAGG-3'; antisense: 5'-CTTTGTCACCGGCCCTTTTG-3'), and $\beta$-actin (sense: 5'-CTTCGCGGGCGACGAT-3'; antisense: 5'-CCACATAGGAATCCTTCTGACC-3'). With $\beta$-actin as a reference, relative LAMC1 mRNA expression level was analyzed using the $2^{-\Delta \Delta \mathrm{Ct}}$ method [15].

3-(4,5-dimethylthiazol-2-yl)-2,5-diphenyltetrazolium bromide (MTT), colony formation, and BrdU assays. MTT, colony formation, and BrdU assays were performed for the detection of cell proliferation. For MTT assay, $1 \times 10^{4}$ AGS and MKN45 cells were plated into 96-well plates. After $48 \mathrm{~h}$, $10 \mu \mathrm{l}$ MTT reagent (Beyotime, Shanghai, China) was added, and cells were incubated for $4 \mathrm{~h}$ at $37^{\circ} \mathrm{C}$. Then the formazan was dissolved using dimethyl sulfoxide (DMSO) (Solarbio), and the absorbance at $570 \mathrm{~nm}$ was measured using a microplate reader (Allsheng, Hangzhou, China). Cell viability was determined by normalizing to the control group.

For colony formation assay, approximately 500 AGS and MKN45 cells were inoculated into 6-well plates. After incubation for 10 days, the clones were stained with $0.1 \%$ crystal violet (Beyotime). Next, the clones were imaged and counted.

For BrdU assay, $5 \times 10^{4}$ AGS and MKN45 cells were added into 24-well plates and cultured for $48 \mathrm{~h}$. Then cells were incubated with $10 \mu \mathrm{M}$ BrdU labeling reagent (Thermo Fisher Scientific) for $4 \mathrm{~h}$. Next, cells were ethanol fixed and incubated with the BrdU antibody (1:50 dilution, Thermo Fisher Scientific) for $30 \mathrm{~min}$, followed by incubation with IgG-fluorescein for another $30 \mathrm{~min}$. BrdU labeled cells were observed under a fluorescence microscope.

Wound healing assay. For migration assay, $2 \times 10^{5}$ AGS and MKN45 cells were seeded into the 6-well plates. When cells were grown to $90 \%$ confluence, a straight wound was made using a pipette tip. Then cells were incubated for $24 \mathrm{~h}$. The wound was recorded at 0 and $24 \mathrm{~h}$ using a microscope. The wound width was detected, and the migratory ability was evaluated according to the relative wound width $(24 \mathrm{~h} / 0 \mathrm{~h})$.

Transwell assay. For invasive ability assay, $1 \times 10^{5} \mathrm{AGS}$ and MKN45 cells in RPMI-1640 medium without serum were added into the Matrigel-coated 24-well Transwell chambers (Corning Inc., Corning, NY, USA). The lower chambers were added with $600 \mu \mathrm{l}$ RPMI-1640 medium plus 10\% FBS. After $24 \mathrm{~h}$, the invasive cells were stained with $0.1 \%$ crystal violet, followed by observation under a magnification $\times 100$ microscope with 3 random fields. The number of invasive cells was determined using Image J software (NIH, Bethesda, MD, USA).

Lactate production, lactate dehydrogenase (LDH) activity, and glucose uptake levels. Lactate production, 
LDH activity, and glucose uptake levels were examined using the specific commercial kits according to the instructions. AGS and MKN45 cells $\left(2 \times 10^{5}\right)$ with the indicated transfection were placed into the 6-well plates for $48 \mathrm{~h}$. Next, the supernatant was harvested, and lactate production, LDH activity, and glucose uptake levels were determined with the Lactate Assay kit, LDH Activity Assay kit, or Glucose Assay kit (Sigma) according to the manufacturers' instructions.

Western blotting. Cells were lysed in radioimmunoprecipitation assay buffer (Beyotime), and protein samples were obtained after centrifugation at $10,000 \times \mathrm{g}$ for $10 \mathrm{~min}$. After the quantification via a bicinchoninic acid kit (Thermo Fisher Scientific), $20 \mu \mathrm{g}$ protein samples were separated onto sodium dodecyl sulfate-polyacrylamide gel electrophoresis, followed by transferring on polyvinylidene difluoride membranes (Solarbio). The membranes were blocked with $3 \%$ bovine serum albumin (BSA) for $1 \mathrm{~h}$, and incubated with primary antibodies for AKT (ab18785, 1:2,000 dilution; Abcam), phosphorylated AKT (p-AKT) (ab38449, 1:500 dilution; Abcam), mitogen-activated protein kinase kinase1 (MEK1) (ab109556, 1:1,000 dilution; Abcam), p-MEK1 (ab96379, 1:3,000 dilution; Abcam), extracellular regulated protein kinase 1/2 (ERK1/2) (ab115799, 1:2,000 dilution; Abcam), p-ERK1/2 (ab223500, 1:300 dilution; Abcam), and $\beta$-actin (ab115777, 1:1,000 dilution; Abcam) overnight, followed by incubated with HRP-labeled secondary antibody IgG (ab97051, 1:10,000 dilution; Abcam) for $2 \mathrm{~h}$. Next, the membranes were exposed to enhanced chemiluminescence solution (Thermo Fisher Scientific) and the blots were visualized. With $\beta$-actin as a loading control, relative protein expression was analyzed using ImageJ v1.8 software.

Statistical analysis. Experiments were repeated three times with three replicates unless otherwise indicated. The data are presented as mean \pm standard deviation (SD). The statistical analysis was processed by SPSS 20.0 (SPSS, Chicago, IL, USA). The correlation between LAMC1 expression and clinicopathological features of patients was analyzed using the $\chi^{2}$ test. The difference between groups was determined by Student's t-test or one-way ANOVA followed via Tukey post hoc test. It was considered significant if $\mathrm{p}<0.05$.

\section{Results}

LAMC1 is highly expressed and associated with the poor prognosis of gastric cancer patients. To determine whether LAMC1 participates in gastric cancer development, its abundance was examined in gastric cancer samples from 42 patients. By comparing with the adjacent normal tissues, LAMC1 abundance was evidently elevated in tumor tissues $(\mathrm{n}=42)$ (Figure 1A). Moreover, the patients were divided into a high or a low expression of the LAMC1 group according to the median of LAMC1 expression ( $n=21$ /group). The high expression of LAMC1 was positively associated with the clinical stage, tumor depth, lymph node metastasis, and distant metastasis of patients (Table 1). Additionally, LAMC1 expression in tumor and normal tissues was further examined via IHC and FISH assays. Results showed the abundance of LAMC1 was elevated in tumor tissues in comparison to normal samples (Figures 1B, 1C). These results suggested the enhanced expression of LAMC1 might implicate in gastric cancer progression and a worse prognosis.

LAMC1 silence represses gastric cancer cell proliferation. To study the function of LAMC1 in gastric cancer progression in vitro, LAMC1 abundance was detected in gastric cancer cell lines. Results indicated a higher LAMC1 expression in AGS, MKN45, SNU-1, and SNU16 cells than the counterpart in GES-1 cells (Figure 2A). Furthermore, AGS and MKN45 cells with relatively higher expression of LAMC1 were chosen for further loss-of-function experiments. AGS and MKN45 cells were transfected with shNC or shLAMC1 (Figure 2B and Supplementary Figure S1). The transfection failed to induce clear morphology change (Supplementary Figure S2). In addition, shLAMC1-2 with the highest knockdown efficacy was referred to as shLAMC1 and used for subsequent functional assays. MTT assay showed LAMC1 knockdown obviously repressed the viability of AGS and MKN45 cells (Figure 2C). Moreover, LAMC1 interference markedly constrained the colony formation ability (Figure 2D). Additionally, LAMC1 was also observed to inhibit DNA replication capacity assessed

Table 1. The association between clinical information of gastric cancer patients and LAMC1 expression.

\begin{tabular}{|c|c|c|c|c|}
\hline Characteristics & $\begin{array}{l}\text { Number of } \\
\text { patients }\end{array}$ & $\begin{array}{c}\text { LAMC1 } \\
\text { Low expression } \\
(<\text { median })\end{array}$ & $\begin{array}{c}\text { LAMC1 } \\
\text { High expression } \\
\text { ( } \geq \text { median })\end{array}$ & p-value \\
\hline Number & 42 & 21 & 21 & \\
\hline Ages (years) & & & & 0.757 \\
\hline$<50$ & 19 & 10 & 9 & \\
\hline$\geq 50$ & 23 & 11 & 12 & \\
\hline Gender & & & & 0.533 \\
\hline Female & 18 & 8 & 10 & \\
\hline Male & 24 & 13 & 11 & \\
\hline Histological type & & & & 0.217 \\
\hline Differentiated & 20 & 12 & 8 & \\
\hline Undifferentiated & 22 & 9 & 13 & \\
\hline Clinical stage & & & & $0.030^{*}$ \\
\hline I-II & 23 & 15 & 8 & \\
\hline III-IV & 19 & 6 & 13 & \\
\hline Tumor depth & & & & $0.013^{*}$ \\
\hline $\mathrm{T} 1-\mathrm{T} 2$ & 20 & 14 & 6 & \\
\hline $\mathrm{T} 3-\mathrm{T} 4$ & 22 & 7 & 15 & \\
\hline \multicolumn{2}{|c|}{ Lymph node metastasis } & & & $0.019^{*}$ \\
\hline N0-N1 & 13 & 10 & 3 & \\
\hline N2-N3 & 29 & 11 & 18 & \\
\hline Distant metastasis & & & & $0.005^{*}$ \\
\hline M0 & 25 & 17 & 8 & \\
\hline M1 & 17 & 4 & 13 & \\
\hline
\end{tabular}

Note: ${ }^{*}$ indicates the significant difference 
A

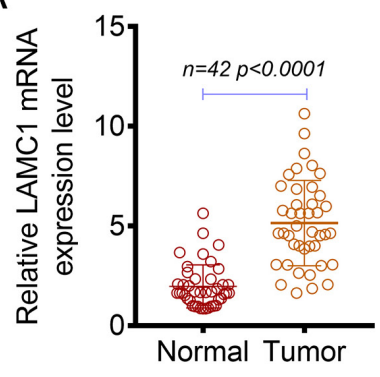

B

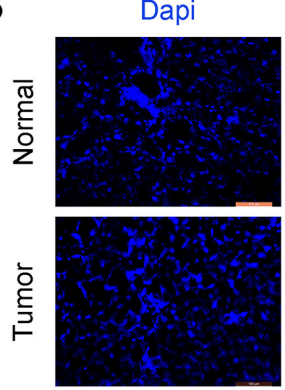

LAMC1
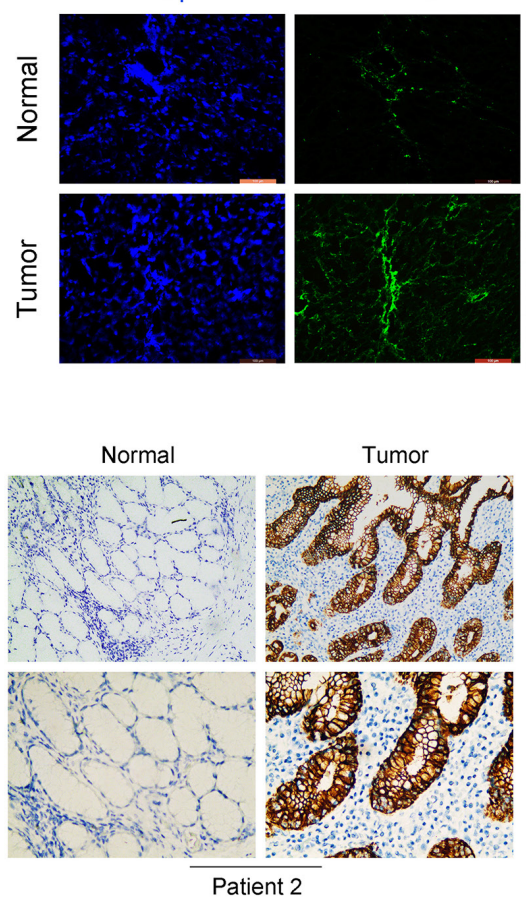

Merge

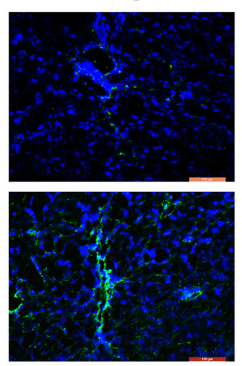

C

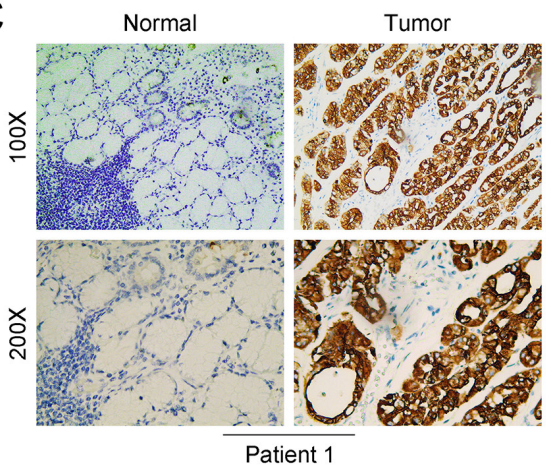

Tumor

Normal

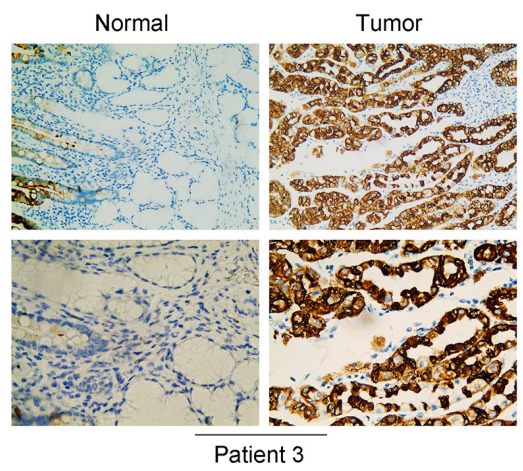

Figure 1. LAMC1 abundance is elevated in gastric cancer tissues. A) LAMC1 abundance in tumor and normal tissues from gastric cancer patients was detected via $\mathrm{qRT}$-PCR, $\mathrm{n=42}$. B) LAMC1 level in tumor and normal tissues was examined via FISH analysis. C) LAMC1 expression in tumor and normal tissues was measured via IHC, $n=3 ; p<0.05$ represents significant difference

A

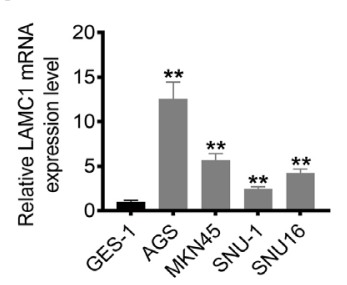

B

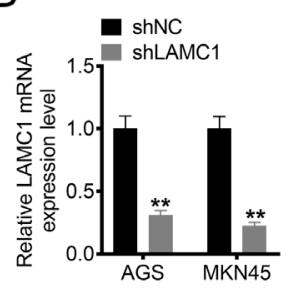

C

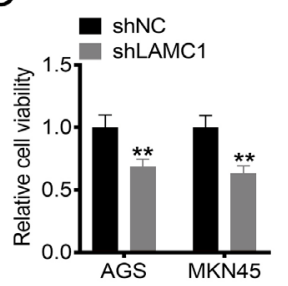

$\mathrm{D}$

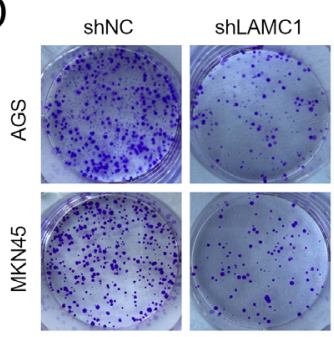

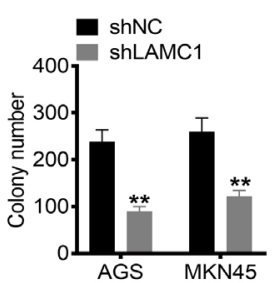

$E$
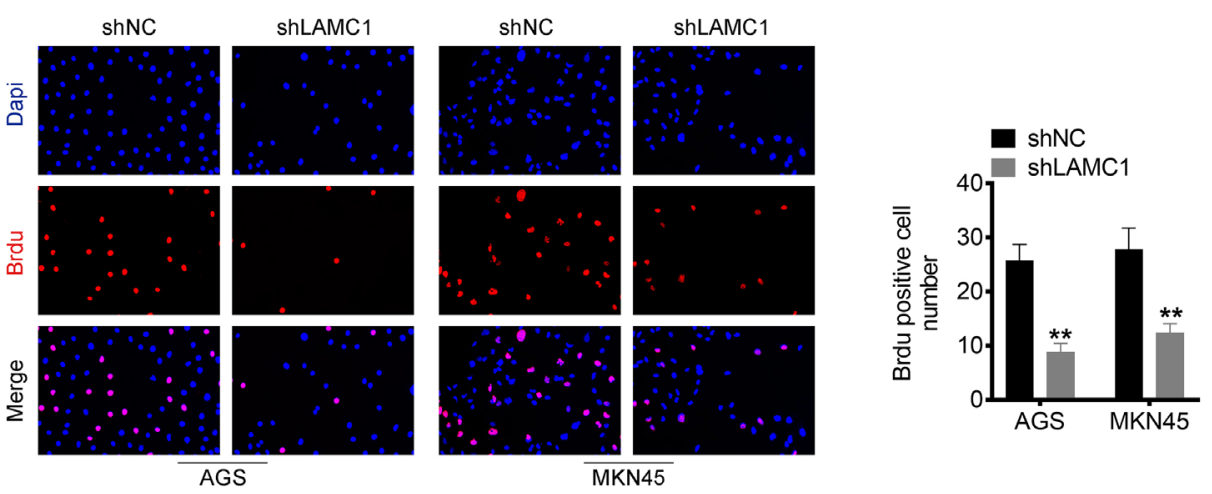

Figure 2. LAMC1 knockdown constrains gastric cancer cell proliferation. A) LAMC1 mRNA level was examined via qRT-PCR in GES-1, AGS, MKN45, SNU-1, and SNU16 cells. B) LAMC1 abundance was examined by qRT-PCR in AGS and MKN45 cells transfected with shNC or shLAMC1. C) Cell viability was measured via MTT in AGS and MKN45 cells transfected with shNC or shLAMC1. D) Colony formation was analyzed in AGS and MKN45 cells transfected with shNC or shLAMC1. E) Cell proliferation was examined via BrdU assay in AGS and MKN45 cells transfected with shNC or shLAMC1. ${ }^{* *} \mathbf{p}<0.01$ 
by the BrdU assay (Figure 2E). These results indicated LAMC1 knockdown played an anti-proliferation role in gastric cancer cells.

LAMC1 knockdown reduces migration and invasion of the gastric cancer cells. The effect of LAMC1 knockdown on cell migration and invasion was further evaluated by wound healing and Transwell assays. The wound-healing assay showed that the LAMC1 depletion significantly restrained cell migration, which was revealed by the wider wound compared with the shNC group (Figure 3A). Moreover, the Transwell assay showed LAMC1 knockdown evidently inhibited cell invasion (Figure $3 \mathrm{~B}$ ). These results suggested the LAMC1 knockdown impaired the migration and invasion capacity of the gastric cancer cells.

LAMC1 interference suppresses the Warburg effect in gastric cancer cells. To evaluate the influence of LAMC1 knockdown on the Warburg effect, lactate production, $\mathrm{LDH}$ activity, and glucose uptake were examined in AGS and MKN45 cells with transfection of shNC or shLAMC1. As shown in Figure 4A, the lactate production was markedly repressed by LAMC1 silence in AGS and MKN45 cells. Moreover, LAMC1 knockdown evidently decreased LDH activity in both cell lines (Figure 4B). Additionally, glucose in medium was higher in the shLAMC1-1 group than the shNC group, suggesting LAMC1 silence significantly restrained glucose uptake (Figure $4 \mathrm{C}$ ). These results indicated LAMC1 silence repressed the Warburg effect in the gastric cancer cells.

LAMC1 knockdown inhibits the activation of AKT and MEK/ERK pathways in gastric cancer cells. To explore the LAMC1 mechanism, the LAMC1-related genes were predicted using LinkedOmics (Supplementary Figure S3A). Based on this database, a total of 888 LAMC1-related genes were identified. Moreover, the KEGG pathway enrichment analysis showed the correlated genes of LAMC1 were mostly enriched in the cGMP-PKG signaling, focal adhesion, vascular smooth muscle contraction, PI3K/AKT signaling pathway, Ras/MEK/ERK signaling pathway, and so on (Supplementary Figure S3B). Given the fact that AKT and MEK/ERK signaling pathways are tightly involved in tumorigenesis, thus, the influence of LAMC1 on AKT and MEK/ ERK pathways was investigated. Results showed that the LAMC1 silencing significantly repressed the phosphorylated levels of AKT, MEK1, and ERK1/2, whereas exhibited little effect on the total of AKT, MEK1, and ERK1/2 (Figure 5). These data suggested that the LAMC1 silencing inhibited the activation of the AKT and MEK/ERK pathway in gastric cancer cells.

A
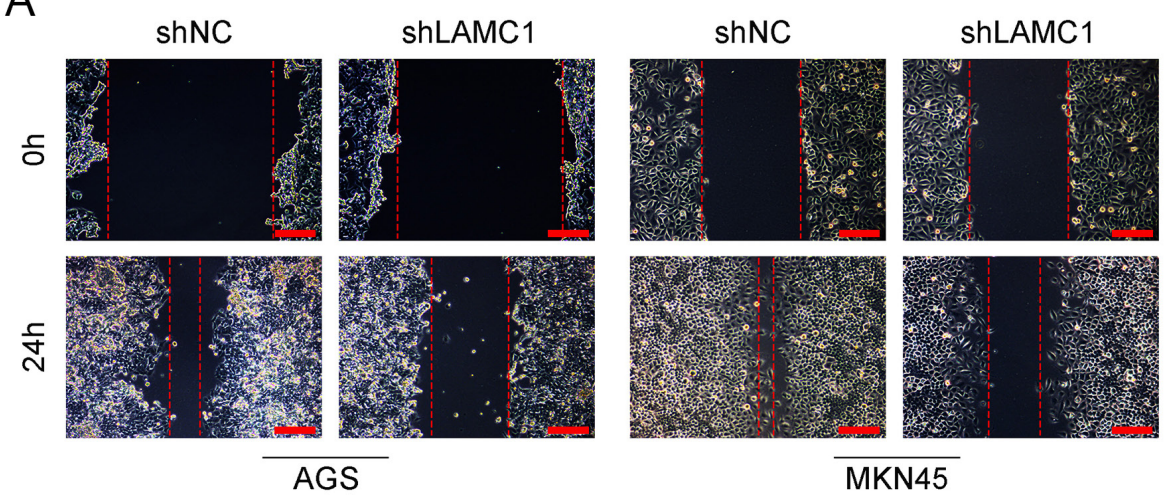

MKN45

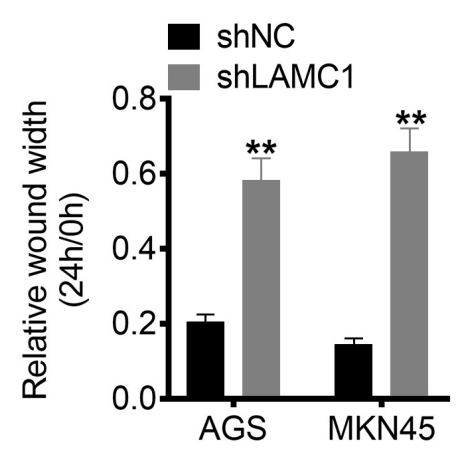

B

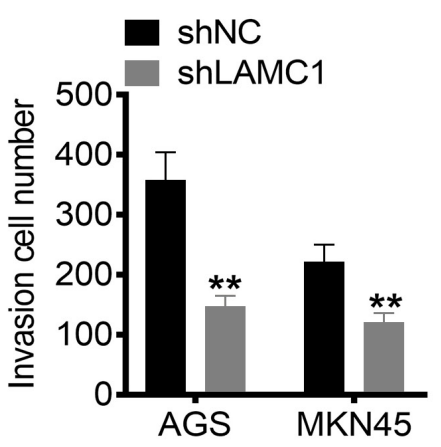

Figure 3. LAMC1 silence restrains gastric cancer cell migration and invasion. A) Cell migration was detected via wound-healing analysis in AGS and MKN45 cells transfected with shNC or shLAMC1. Scale bars: $200 \mu \mathrm{m} \mathrm{B}$ ) Cell invasion was measured via Transwell analysis in AGS and MKN45 cells transfected with shNC or shLAMC1. Scale bars: $200 \mu \mathrm{m} ;{ }^{\star *} \mathrm{p}<0.01$ 
A

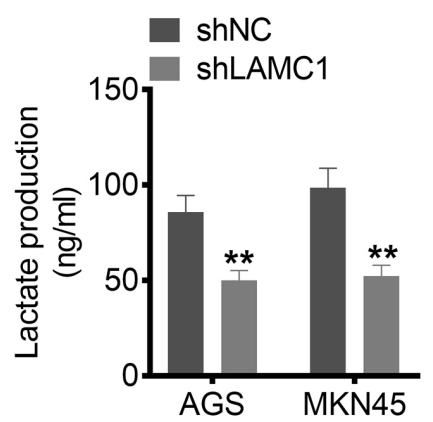

B

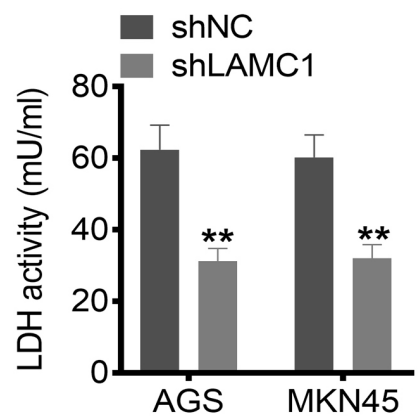

C

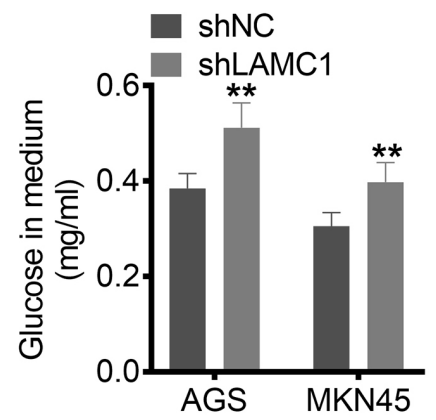

Figure 4. LAMC1 knockdown represses the Warburg effect in gastric cancer cells. Lactate production (A), LDH activity (B), and glucose uptake (C) were detected in AGS and MKN45 cells transfected with shNC or shLAMC1. ${ }^{*} \mathrm{p}<0.05$
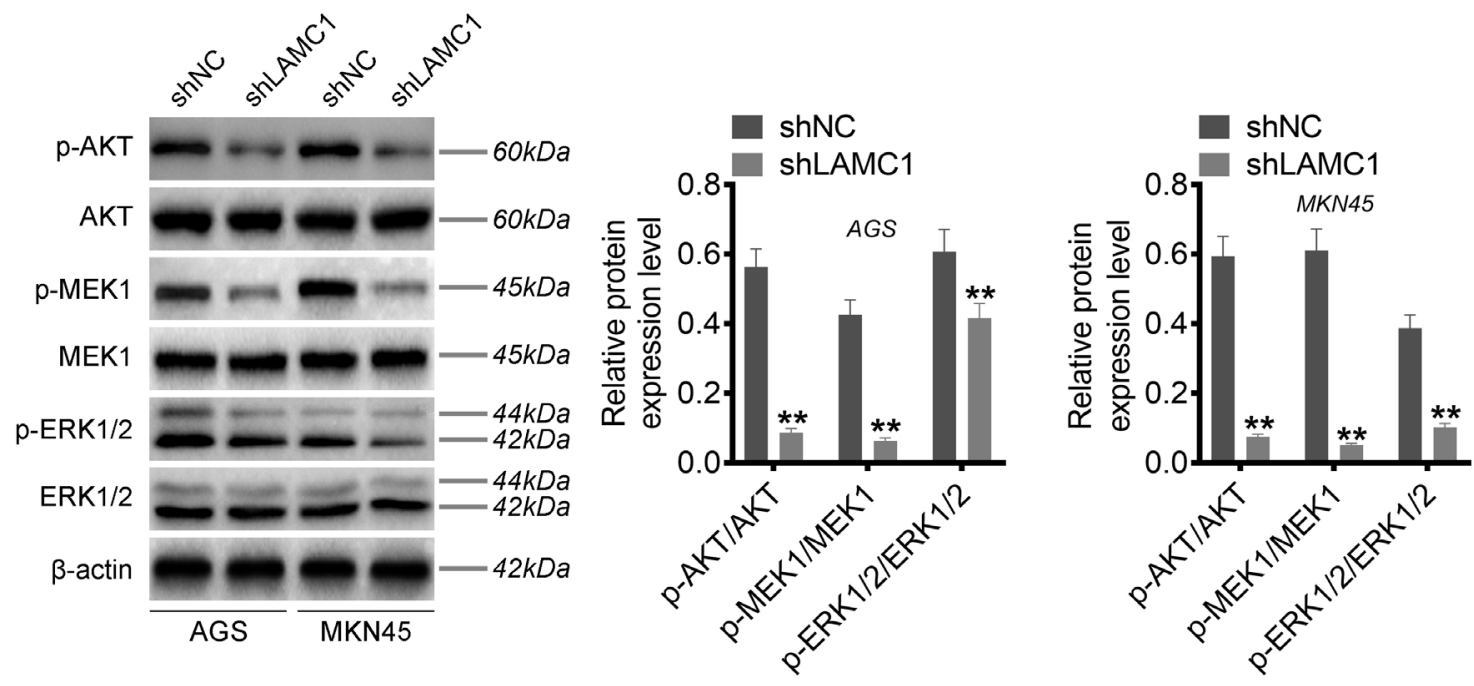

Figure 5. LAMC1 inhibition decreases the activation of AKT and MEK/ERK pathways in gastric cancer cells. The protein levels of p-AKT, AKT, pMEK1, MEK1, p-ERK1/2, and ERK1/2 were detected via western blotting in AGS and MKN45 cells transfected with shNC or shLAMC1. ${ }^{\star *}$ p $<0.01$

\section{Discussion}

Gastric cancer is global cancer, which contributes to $\sim 8.2 \%$ of cancer-related deaths [16]. Although the diagnosis, prevention, and treatment of gastric cancer have improved, the outcomes of patients remain unsatisfactory [17]. Here we first validated that LAMC1 could serve as a prognostic factor for gastric cancer, and our further experiments revealed the anti-cancer activity of LAMC1 knockdown in gastric cancer progression.

An increasing number of studies indicated that LAMC1 predicted the poor prognosis of patients in various tumors, such as endometrial cancer, hepatocellular carcinoma, glioma, and head and neck squamous cell carcinoma $[8$, $9,18,19]$. Similarly, our study firstly demonstrated the increased expression of LAMC1 related to the clinical stage, tumor depth, lymph node metastasis, and distant metas- tasis of patients, suggesting the prognostic value of LAMC1 in gastric cancer. A previous study reported LAMC1 acted as a target of microRNA-29s to promote cell migration and invasion in prostate cancer [10]. Moreover, as a target of microRNA-506 or microRNA-124, LAMC1 could facilitate cell proliferation, migration, and invasion in colorectal and hepatocellular carcinoma [20, 21]. Additionally, LAMC1 knockdown was validated to inhibit cell proliferation and the Warburg effect via regulating the activity of pyruvate kinase type M2 (PKM2) through the phosphatase and tensin homolog (PTEN)/AKT pathway [11]. These all suggested that LAMC1 might play an oncogenic function in human cancers via increasing cell proliferation, migration, invasion, and the Warburg effect. Consistent with these reports, our study also found the inhibitive effect of LAMC1 inhibition on tumorigenesis of gastric cancer, indicating LAMC1 might serve as an important target for gastric cancer treatment. 
The AKT pathway is a poor prognostic factor and contributes to the malignant progression in gastric cancer [22, 23]. The inhibitors targeting the AKT signaling have the potential for therapy of gastric cancer [24]. It is well established that the AKT pathway contributed to cell proliferation, migration, and invasion in gastric cancer [25-27]. Furthermore, the AKT pathway was also observed to participate in the Warburg effect in gastric cancer $[28,29]$. Hence, we explored and confirmed that LAMC1 could affect the AKT pathway in gastric cancer, which agreed with the previous studies on other malignancies $[11,30]$. Apart from the AKT signaling, the MEK/ERK pathway was also involved in the malignant phenotypes of gastric cancer [31-33]. However, whether LAMC1 could interact with the MEK/ERK pathway in gastric cancer is unknown. Here we first identified LAMC1 activated the MEK/ERK pathway and the anti-tumor role of LAMC1 knockdown in gastric cancer may via the deactivation of the AKT and MEK/ERK pathways.

However, there were some limitations in the current research. The direct interaction between LAMC1 and AKT and MEK/ERK pathway was not well illustrated, which would be explored in our future study. Moreover, the microenvironment in vitro was different from that in vivo. Thus, the knockdown of LAMC1 in tumorigenesis in vivo should be further investigated.

In conclusion, LAMC1 knockdown restrained cell proliferation, migration, invasion, and the Warburg effect in gastric cancer, possibly via blocking the activation of the AKT and MEK/ERK pathways. This study indicates a new insight into the pathogenesis of gastric cancer and provides a novel target for the treatment of gastric cancer.

Supplementary information is available in the online version of the paper.

\section{References}

[1] SMYTH EC, NILSSON M, GRABSCH HI, VAN GRIEKEN NC, LORDICK F. Gastric cancer. Lancet 2020; 396: 635-648. https://doi.org/10.1016/S0140-6736(20)31288-5

[2] FIGUEIREDO C, CAMARGO MC, LEITE M, FUENTES-PANANA EM, RABKIN CS et al. Pathogenesis of Gastric Cancer: Genetics and Molecular Classification. Curr Top Microbiol Immunol 2017; 400: 277-304. https://doi. org/10.1007/978-3-319-50520-6_12

[3] ILSON DH. Advances in the treatment of gastric cancer. Curr Opin Gastroenterol 2018; 34: 465-468. https://doi. org/10.1097/MOG.0000000000000475

[4] MATSUOKA T, YASHIRO M. Biomarkers of gastric cancer: Current topics and future perspective. World J Gastroenterol 2018; 24: 2818-2832. https://doi.org/10.3748/wjg.v24. i26.2818

[5] YAO Y. Laminin: loss-of-function studies. Cell Mol Life Sci 2017; 74: 1095-1115. https://doi.org/10.1007/s00018-016$2381-0$
[6] WANG X, HOU Q, ZHOU X. LAMA4 expression is activated by zinc finger Eboxbinding homeobox 1 and independently predicts poor overall survival in gastric cancer. Oncol Rep 2018; 40: 1725-1733. https://doi.org/10.3892/ or.2018.6564

[7] II M, YAMAMOTO H, TANIGUCHI H, ADACHI Y, NAKAZAWA $\mathrm{M}$ et al. Co-expression of laminin beta3 and gamma2 chains and epigenetic inactivation of laminin alpha3 chain in gastric cancer. Int J Oncol 2011; 39: 593-599. https://doi.org/10.3892/ijo.2011.1048

[8] ZHANG Y, XI S, CHEN J, ZHOU D, GAO H et al. Overexpression of LAMC1 predicts poor prognosis and enhances tumor cell invasion and migration in hepatocellular carcinoma. J Cancer 2017; 8: 2992-3000. https://doi.org/10.7150/ jca. 21038

[9] KUNITOMI H, KOBAYASHI Y, WU RC, TAKEDA T, TOMINAGA E et al. LAMC1 is a prognostic factor and a potential therapeutic target in endometrial cancer. J Gynecol Oncol 2020; 31: e11. https://doi.org/10.3802/jgo.2020.31.e11

[10] NISHIKAWA R, GOTO Y, KOJIMA S, ENOKIDA H, CHIYOMARU T et al. Tumor-suppressive microRNA-29s inhibit cancer cell migration and invasion via targeting LAMC1 in prostate cancer. Int J Oncol 2014; 45: 401-410. https://doi. org/10.3892/ijo.2014.2437

[11] YE G, QIN Y, WANG S, PAN D, XU S et al. Lamc1 promotes the Warburg effect in hepatocellular carcinoma cells by regulating PKM2 expression through AKT pathway. Cancer Biol Ther 2019; 20: 711-719. https://doi.org/10.1080/15384047.2 018.1564558

[12] VASAIKAR SV, STRAUB P, WANG J, ZHANG B. LinkedOmics: analyzing multi-omics data within and across 32 cancer types. Nucleic Acids Res 2018; 46: D956-D963. https:// doi.org/10.1093/nar/gkx1090

[13] KANEHISA M, GOTO S. KEGG: kyoto encyclopedia of genes and genomes. Nucleic Acids Res 2000; 28: 27-30. https://doi.org/10.1093/nar/28.1.27

[14] DENNIS G JR, SHERMAN BT, HOSACK DA, YANG J, GAO W et al. DAVID: Database for Annotation, Visualization, and Integrated Discovery. Genome Biol 2003; 4: P3.

[15] LIVAK KJ, SCHMITTGEN TD. Analysis of relative gene expression data using real-time quantitative PCR and the 2(-Delta Delta C(T)) Method. Methods 2001; 25: 402-408. https://doi.org/10.1006/meth.2001.1262

[16] THRIFT AP, EL-SERAG HB. Burden of Gastric Cancer. Clin Gastroenterol Hepatol 2020; 18: 534-542. https://doi. org/10.1016/j.cgh.2019.07.045

[17] LYONS K, LE LC, PHAM YT, BORRON C, PARK JY et al. Gastric cancer: epidemiology, biology, and prevention: a mini review. Eur J Cancer Prev 2019; 28: 397-412. https:// doi.org/10.1097/CEJ.0000000000000480

[18] LIU J, LIU D, YANG Z, YANG Z. High LAMC1 expression in glioma is associated with poor prognosis. Onco Targets Ther 2019; 12: 4253-4260. https://doi.org/10.2147/OTT. S205333

[19] JIANG P, HE S, LI Y, XU Z. Identification of Therapeutic and Prognostic Biomarkers of Lamin C (LAMC) Family Members in Head and Neck Squamous Cell Carcinoma. Med Sci Monit 2020; 26: e925735. https://doi.org/10.12659/ MSM.925735 
[20] ZU C, LIU T, ZHANG G. MicroRNA-506 Inhibits Malignancy of Colorectal Carcinoma Cells by Targeting LAMC1. Ann Clin Lab Sci 2016; 46: 666-674.

[21] YANG ZP, MA HS, WANG SS, WANG L, LIU T. LAMC1 mRNA promotes malignancy of hepatocellular carcinoma cells by competing for MicroRNA-124 binding with CD151. IUBMB Life 2017; 69: 595-605. https://doi.org/10.1002/ iub. 1642

[22] CAO F, ZHANG C, HAN W, GAO XJ, MA J et al. p-Akt as a potential poor prognostic factor for gastric cancer: a systematic review and meta-analysis. Oncotarget 2017; 8: 59878-59888. https://doi.org/10.18632/oncotarget.17001

[23] KANG BW, CHAU I. Molecular target: pan-AKT in gastric cancer. ESMO Open 2020; 5: e000728. https://doi. org/10.1136/esmoopen-2020-000728

[24] SINGH SS, YAP WN, ARFUSO F, KAR S, WANG C et al. Targeting the PI3K/Akt signaling pathway in gastric carcinoma: A reality for personalized medicine? World J Gastroenterol 2015; 21: 12261-12273. https://doi.org/10.3748/wjg. v21.i43.12261

[25] LIU Y, HUANG Y, ZHANG J, PEI C, HU J et al. TIMMDC1 Knockdown Inhibits Growth and Metastasis of Gastric Cancer Cells through Metabolic Inhibition and AKT/GSK3beta/beta-Catenin Signaling Pathway. Int J Biol Sci 2018; 14: 1256-1267. https://doi.org/10.7150/ijbs.27100

[26] HUANG Z, LIANG H, CHEN L. RAB43 Promotes Gastric Cancer Cell Proliferation and Metastasis via Regulating the PI3K/AKT Signaling Pathway. Onco Targets Ther 2020; 13: 2193-2202. https://doi.org/10.2147/OTT.S237356
[27] ZHANG Y, CHEN L, CAO Y, CHEN S, XU C et al. LETM1 Promotes Gastric Cancer Cell Proliferation, Migration, and Invasion via the PI3K/Akt Signaling Pathway. J Gastric Cancer 2020; 20: 139-151. https://doi.org/10.5230/jgc.2020.20.e12

[28] LIU J, PAN C, GUO L, WU M, GUO J et al. A new mechanism of trastuzumab resistance in gastric cancer: MACC1 promotes the Warburg effect via activation of the PI3K/AKT signaling pathway. J Hematol Oncol 2016; 9: 76. https://doi. org/10.1186/s13045-016-0302-1

[29] WU J, ZHANG X, WANG Y, SUN Q, CHEN M et al. Licochalcone A suppresses hexokinase 2-mediated tumor glycolysis in gastric cancer via downregulation of the Akt signaling pathway. Oncol Rep 2018; 39: 1181-1190. https://doi. org/10.3892/or.2017.6155

[30] HE R, KONG Y, FANG P, LI L, SHI H et al. Integration of quantitative proteomics and metabolomics reveals tissue hypoxia mechanisms in an ischemic-hypoxic rat model. J Proteomics 2020; 228: 103924. https://doi.org/10.1016/j. jprot.2020.103924

[31] ZHAO Y, HE J, LI Y, XU M, PENG X et al. PHF14 Promotes Cell Proliferation and Migration through the AKT and ERK1/2 Pathways in Gastric Cancer Cells. Biomed Res Int 2020; 2020: 6507510. https://doi.org/10.1155/2020/6507510

[32] LIU D, XIAO P, FENG C, MENG H, BI E. Long non-coding RNA GASL1 restrains gastric carcinoma cell proliferation and metastasis by sponging microRNA-106a. Cell Cycle 2020; 19: 2611-2621. https://doi.org/10.1080/15384101.202 0.1812918

[33] SUN L, CHEN G, SUN A, WANG Z, HUANG H et al. BAG2 Promotes Proliferation and Metastasis of Gastric Cancer via ERK1/2 Signaling and Partially Regulated by miR186. Front Oncol 2020; 10: 31. https://doi.org/10.3389/fonc.2020.00031 
https://doi.org/10.4149/neo_2021_201117N1239

\section{LAMC1 is related to the poor prognosis of patients with gastric cancer and facilitates cancer cell malignancies}

Zhi-Rong HAN*, Xiao-Lin JIANG, Wen-Chuan FAN

Supplementary Information

A

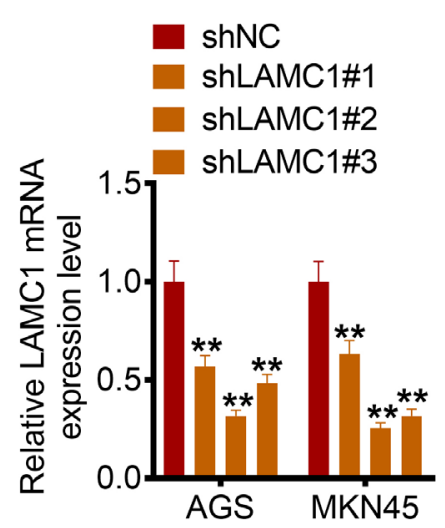

B

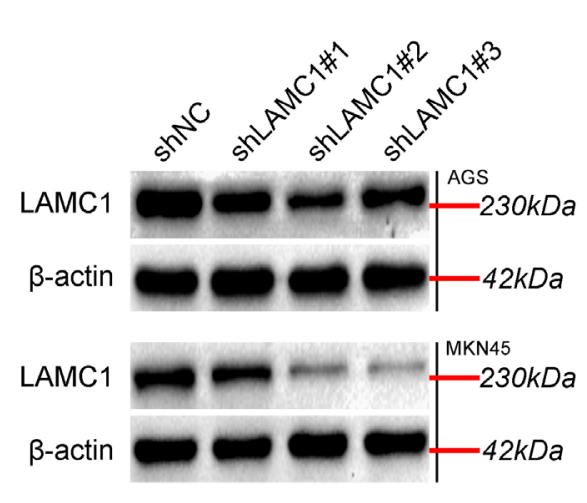

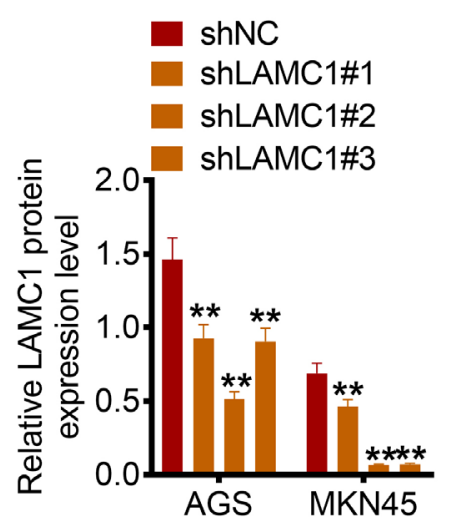

Supplementary Figure S1. Knockdown efficiency of different LAMC1 shRNAs. mRNA (A) and protein (B) levels of LAMC1 in AGS and MKN45 cells after transfected with different shRNAs targeting LAMC1. ${ }^{* *} \mathrm{p}<0.01$
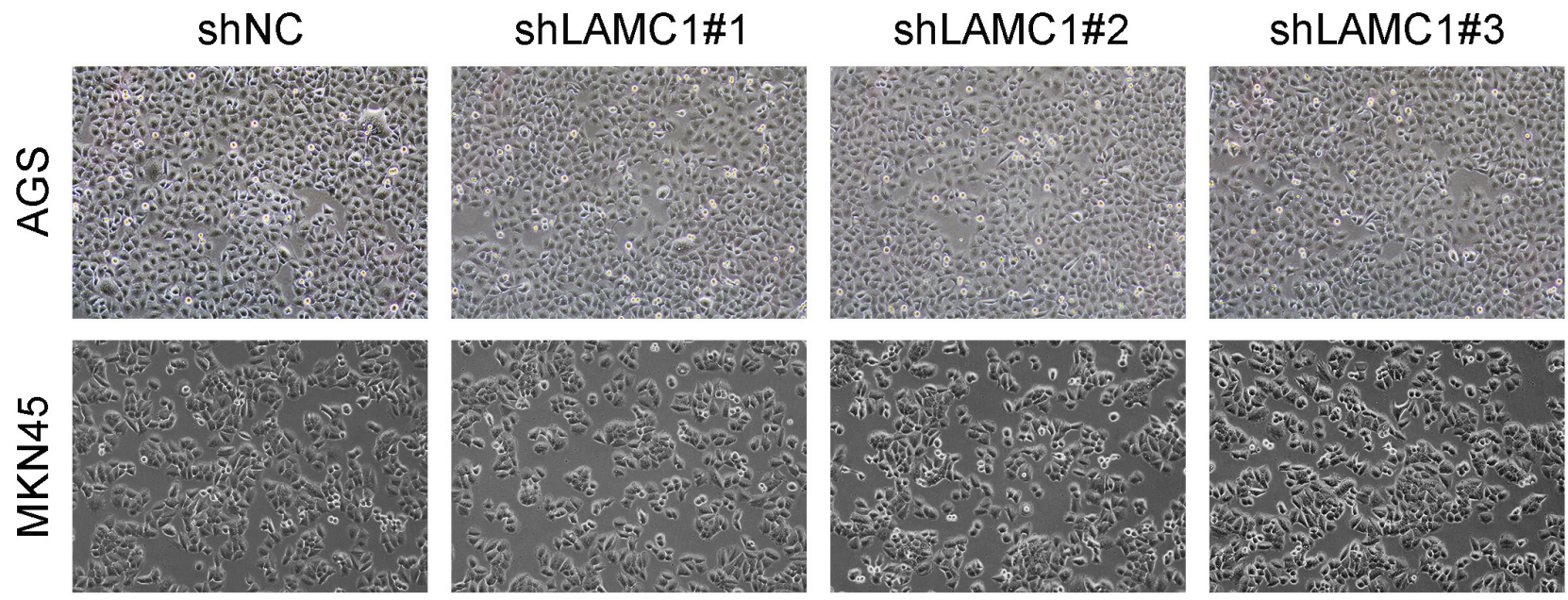

Supplementary Figure S2. Cell morphology observed under light microscope. AGS and MKN45 cells were transfected with different shRNAs targeting LAMC1 after $48 \mathrm{~h}$. 
A

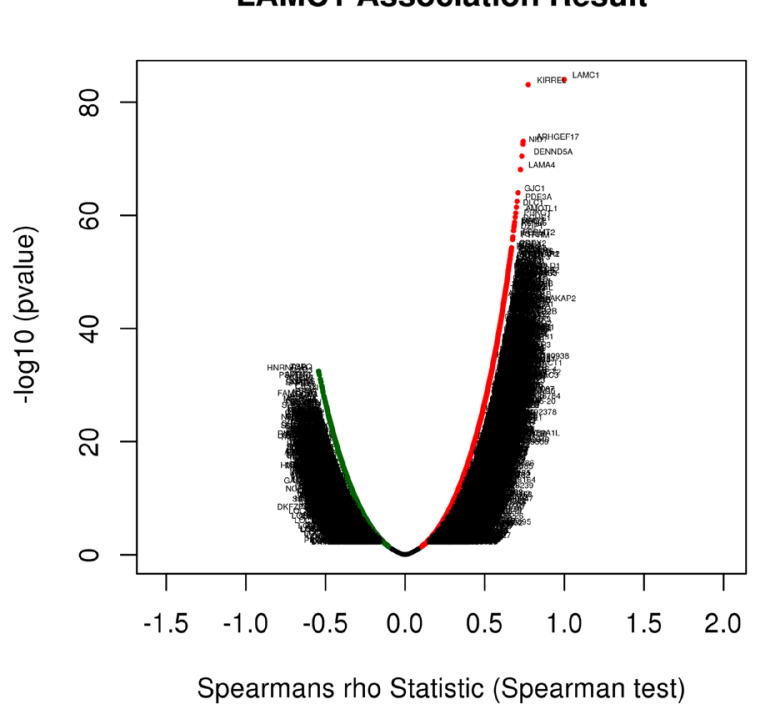

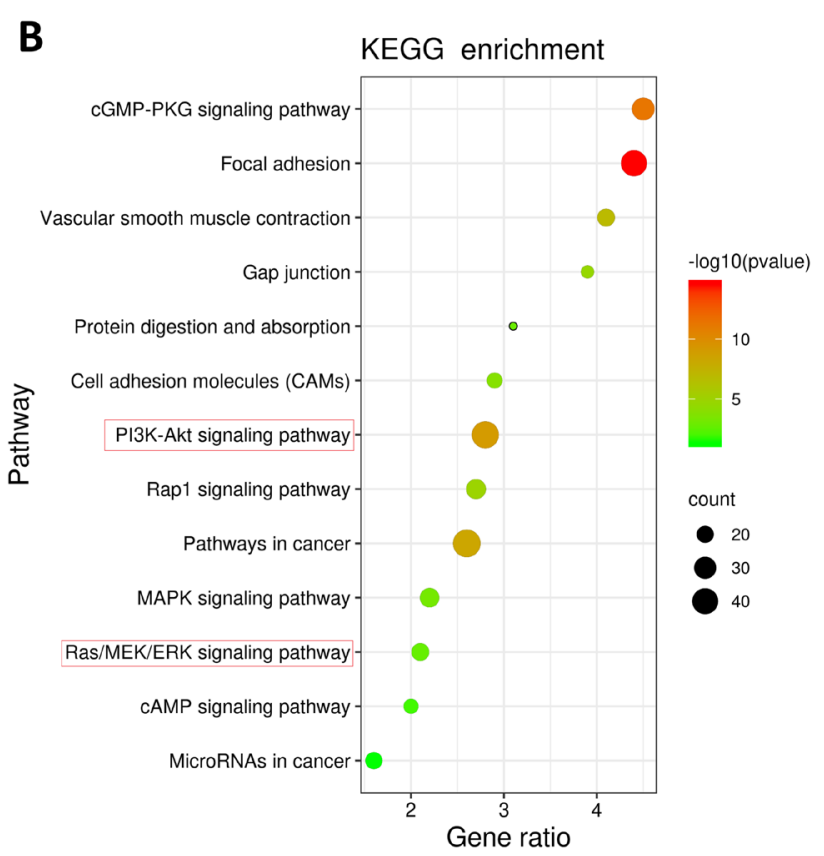

Supplementary Figure S3. LAMC1-related genes in gastric cancer were predicted using LinkedOmics (A). These genes were analyzed by KEGG pathway enrichment analysis using DAVID tool (B). 